\title{
Development of a wearable assistive soft robotic device for elbow rehabilitation
}

Conference or Workshop Item

Accepted Version

Oguntosin, V., Harwin, W. S., Kawamura, S., Nasuto, S. J. and Hayashi, Y. (2015) Development of a wearable assistive soft robotic device for elbow rehabilitation. In: The 2015 ICORR 11th International Conference on Rehabilitation Robotics, 1114 Aug 2015, Singapore, pp. 747-752. Available at http://centaur.reading.ac.uk/40674/

It is advisable to refer to the publisher's version if you intend to cite from the work. See Guidance on citing.

Published version at: http://dx.doi.org/10.1109/ICORR.2015.7281291

All outputs in CentAUR are protected by Intellectual Property Rights law, including copyright law. Copyright and IPR is retained by the creators or other copyright holders. Terms and conditions for use of this material are defined in the End User Agreement. 


\section{www.reading.ac.uk/centaur}

\section{CentAUR}

Central Archive at the University of Reading

Reading's research outputs online 


\title{
Development of a Wearable Assistive Soft Robotic Device for Elbow Rehabilitation
}

\author{
Victoria Oguntosin, William S. Harwin, Sadao Kawamura, Slawomir J. Nasuto and Yoshikatsu Hayashi
}

\begin{abstract}
The loss of motor function at the elbow joint can result as a consequence of stroke. Stroke is a clinical illness resulting in long lasting neurological deficits often affecting somatosensory and motor cortices. More than half of those that recover from a stroke survive with disability in their upper arm and need rehabilitation therapy to help in regaining functions of daily living. In this paper, we demonstrated a prototype of a low-cost, ultra-light and wearable soft robotic assistive device that could aid administration of elbow motion therapies to stroke patients. In order to assist the rotation of the elbow joint, the soft modules which consist of soft wedge-like cellular units was inflated by air to produce torque at the elbow joint. Highly compliant rotation can be naturally realised by the elastic property of soft silicone and pneumatic control of air. Based on the direct visual-actuation control, a higher control loop utilised visual processing to apply positional control, the lower control loop was implemented by an electronic circuit to achieve the desired pressure of the soft modules by Pulse Width Modulation. To examine the functionality of the proposed soft modular system, we used an anatomical model of the upper limb and performed the experiments with healthy participants.
\end{abstract}

\section{INTRODUCTION}

Stroke results from damage to the vascular supply of the brain resulting in neuronal death in the brain [1]. Therefore, stroke patients have difficulty performing specific motions such as elbow flexion and extension due to damage of the motor and somatosensory cortices. Early muscle activation is critical to good recovery [2], hence the need for exercises [1], [3]. Stroke neuro-rehabilitation is therefore important to restore muscle functions to the damaged body parts and help stroke surviving patients regain the ability to perform activities of daily living. This rehabilitation is required especially during the early stage of stroke diagnosis when there is a greater opportunity to influence neural plasticity and brain recovery [4]. Stroke rehabilitation requires long, manipulative and intensive direct physical therapy sessions to improve strength, accuracy, and range of motion [5]. Recent research work on robot therapies have been shown to be safe and beneficial over human delivered therapies due to their flexibility and intensity [6]. An inexpensive, compliant, wearable and lightweight robotic device allowing patients to engage in exercises on their own, either at home or in

Victoria Oguntosin, William S. Harwin, Slawomir J. Nasuto and Yoshikatsu Hayashi are with Systems Neuroscience Group, School of Systems Engineering, University of Reading, U.K. Sadao Kawamura is with Department of Robotics, Ritsumeikan University, Japan. Email: v.w.oguntosin@pgr.reading.ac.uk; w.s.harwin@ reading.ac.uk; s.j.nasuto@reading.ac.uk; y.hayashi@reading.ac.uk the hospital, would make physical therapy more available to patients [3], [7].

There has been a growing interest in using soft actuators for rehabilitation training, for example, the McKibben pneumatic artificial muscles are the most highly developed and studied class of soft actuators [8], [9]. State of the art in rehabilitation soft robotics includes lower limb gait rehabilitation [10] to facilitate hip movement for a rodent with spinal cord injury, a rehabilitation glove to achieve flexing motion for the thumb [11] and human fingers [7] by generating sufficient amount of speed, range of motion and forces for these motions. Fluidic driven upper limb orthosis have been used for rehabilitation [12]. Soft robots are predominantly made of continuously deformable high-strain rubber [13]. This feature makes them inherently soft and therefore safe for direct attachment to the skin. Pneumatic actuation is often required to generate assistive torques to joints of the body. Soft robots have been shown to deliver motions ranging from single degree-of-freedom movement such as bending and angular displacement to multiple modes of gait [14], [15], [16], [17]. The ease of fabrication and low cost of manufacture and actuation [18] enables actuator geometry design and production in a short time.

In contrast to conventional assistive devices [19], [20] which are made of rigid heavy metal, and as a result, require the constrain of the body trunk, we aim at wearable and lightweight assistive modules which can provide appropriate degrees of assistive force to the elbow. The lightweight modules made of soft inflatable structures allow us to provide assistive force while ensuring safe human-robotic interaction [8] required for neuro-rehabilitation. In contrast to the stateof-the art of soft robots currently used to provide assistive motion, our design utilises a low air pressure supply of $50 \mathrm{KPa}$, with highly compliant silicone rubber having shore A hardness. The cost of the complete soft robotic device including the actuation system is extremely low and very affordable.

We describe the design of an entirely soft and inexpensive robot, composed of $100 \%$ silicone rubber which offers upper limb assistance for the elbow. The actuator is designed to be wearable around the arm and forearm using silicone bands as attachment thereby demonstrating its safety, ease of fitting and removal as well as its practicability as a physiotherapy apparatus. The main contribution of this paper is the construction and testing of an entirely soft, light-weight, one degree of freedom prototype elbow joint rehabilitation 
training system. This system offers upper limb assistance by providing for elbow flexion and extension. Pneumatic control of air in the soft modules is achieved by using a pressure sensor as feedback while the angle of motion was obtained using visual information from a webcam.

\section{DESIGN CONSIDERATIONS}

During the intensive therapies of the upper limb delivered to stroke patients by trained physiotherapist, muscles are stretched and the elbow joint is flexed and extended through its range of motion in order to recover the smooth and elastic functions of muscles. Hard robotic structures are mostly used to produce these motions. They are based on rigid links connected by joints - this makes them heavy with expensive and complicated control. Furthermore, they tend to be supported by a solid base on the ground. However, this kind of constraint largely restricts the horizon of assistive devices. Moreover, hard assistive devices require appropriate gravity compensation in proportion to the weight of the upper limb to offset gravity effects. All of these considerations lead to a set of design specifications required to be fulfilled by a soft wearable robotic assistive device in order to successfully assist in elbow motion.

1) Visco-elastic property for passive motion: To realise ideal trajectories, positional control is applied, and the amount of force to restore the desired trajectory should be controlled compliantly by visco-elastic property of the soft actuator.

2) Structural transparency for active motion: The structural transparency is such that the mass of the actuator is so small that a patient performing spontaneous motion does not feel resistance in interactions with the robotic arm. An actuator mass less than $0.5 \mathrm{~kg}$ is desirable [7].

3) Wearable assistive robot: It should not require the base coordinate system of robot to be grounded so that it does not constrain the motion of the main body.

A soft assistive device should be able to deliver appropriate assistive torque for the particular motion according to the torque normally required for a typical healthy individual. The production of an appropriate assistive torque for rotational motion can be difficult due to inherent softness and its ability to generate a fraction of the normal assistive torque produced by conventional robots.

Our design is such that soft wedge-like inflatable modules are actuated to produce torque. The measured maximum torque for this actuator is $2.7 \mathrm{Nm}$, the typical human isometric strength for elbow flexion/extension is $1.2 \mathrm{Nm}$ [21] which proves the viability of the soft modules to assist in these motions. When worn on the upper limb and actuated via inflation, a change in wedge angle would result which acts to generate a rotational motion, thereby assisting the movement of the elbow for extension and flexion while the control of air pressure in individual modules will contribute to the compliant assistance. Other specifications such as intrinsic safety; range of motion; comfort of wearing as well as extremely low manufacturing cost was implemented.

\section{ACTUATOR DESIGN AND FABRICATION}

\section{A. Actuator Geometrical Parameters}

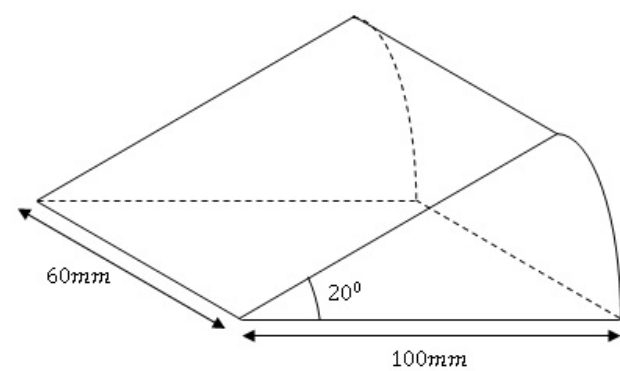

Fig. 1: Schematic of a single soft module.

In this study, a number of geometrical parameters in the design of the soft assistive device can be identified as shown in Figure 1. These parameters are defined by the unactuated angle of the actuator, base length, height of air channel, width of the actuator and wall thickness.

TABLE I: Basic Characteristics of the Rehabilitation Soft Modules

\begin{tabular}{|l|l|}
\hline Characteristics & Value \\
\hline Weight & $0.35 \mathrm{Kg}$ \\
\hline Degrees of Freedom & 1 (Elbow Flexion and Extension) \\
\hline Assistive Torque & $2.7 \mathrm{Nm}$ \\
\hline Range of Motion & $90^{\circ}-130^{\circ}$ \\
\hline Operating Pressure & $0-50 \mathrm{KPa}$ \\
\hline Specified Purpose & Elbow Rehabilitation \\
\hline Safety factors & $\begin{array}{l}\text { Composed of } 100 \% \text { silicone } \\
\text { Ease of wearing }\end{array}$ \\
\hline
\end{tabular}

This rehabilitation actuator is composed of four wedgeshaped segment having an individual angle of $20^{\circ}$. The opposite layers of the $20^{\circ}$ angle are made of highly inextensible silicone and the other layers are casted with highstrain silicone. These layers direct the structure to increase the angle between the top and the bottom layers as the actuator is pressurised; and decrease its angle when depressurised. Angular displacement is therefore achieved by pneumatically pressurizing/depressurizing the embedded air channels within the soft modules. When pressurised, the channels undergo high strain and effectively increase in angle. Because of the strain-limiting layers, this expansion generates angular displacement about the stiffer layer. This design is modular by enabling scalability in the number of wedge-shaped segments. Table I shows a summary of the design characteristics of the soft modules.

\section{B. Fabrication Process of Soft Actuator}

The wedge-shaped modules were produced by a two-part casting process. The geometry of the mould for the soft actuator was designed using SolidWorks, a 3D CAD design software. The mould is then printed out with Acrylonitrile 


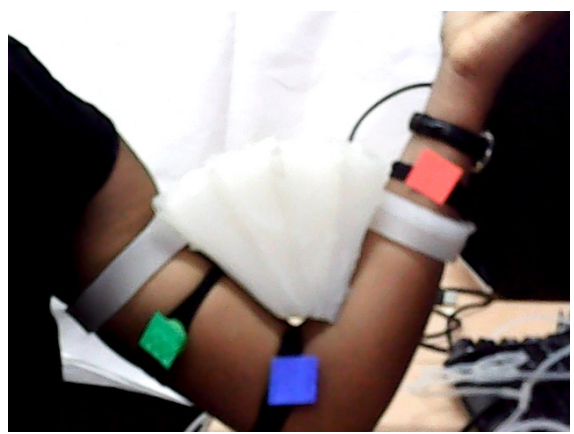

(a)

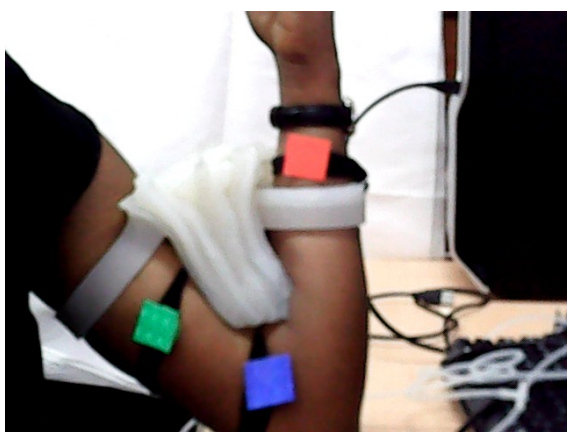

(b)

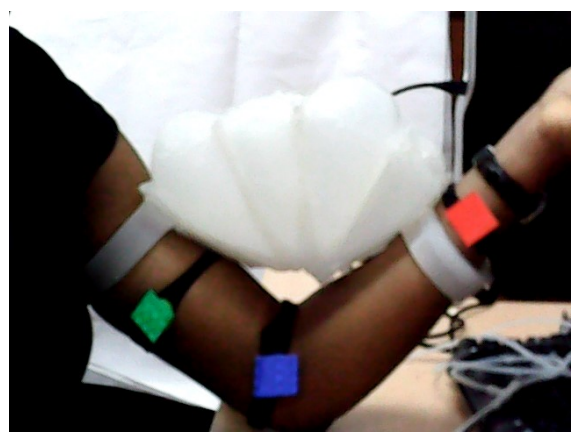

(c)

Fig. 2: Soft modules worn by a healthy subject. The red, blue and green colour markers were used by the visual processing system to measure the current angle of the elbow. a) the soft modules in an unactuated state b) the soft modules are depressuzied at a vacuum pressure of $-10 \mathrm{KPa}$ with the elbow in flexion $\mathrm{c}$ ) the soft modules pressurised at a pressure of $7 \mathrm{KPa}$ with the elbow in an extended state.

Butadiene Styrene (ABS) plastic using HP DeskJet $($ $3 \mathrm{D}$ printer. Ecoflex 0030 silicone with a viscousity of 3000cPs was used as the highly-extensible layer while Addition Cure 33 silicone with a viscousity of $7000 \mathrm{cPs}$ was used as the low strain layer. Both grades of silicone liquid comes in two parts - Parts A and B. Both parts were mixed in equal quantity (by weight) and poured into the $3 \mathrm{D}$ printed moulds, and allowed to cure at room temperature for 4 hours.

The main body of the actuator, composed of the softer cured silicone is then immersed into the base mould containing the high-stiffness liquid silicone to create a perfect seal with the bottom layer after curing. These two layers (soft main body and hard base) produces a perfect seal because cured silicone bonds sufficiently well with liquid silicone after curing. After the two layers are bonded together, a puncture size of about $2 \mathrm{~mm}$ is created to make a tube opening into the air channel; silicone tubing is then inserted into the air channel. Lastly, the contact point of the structure and tubing are sealed with a final coating of silicone to minimise air leakage. Repeating these fabrication procedures, four of these same actuators were produced. They were glued together with fine coating of liquid silicone to produce the final design.

\section{Design of Attachment}

Attachments of the soft modules to the human upper limb were designed to facilitate large area of contact, stable fit, comfort of wearing and removal. Attachment was required to provide a smooth distribution of contact pressure over the entire length of the arm and forearm thereby minimizing discomfort and provide a soft but secure attachment. These were taken into consideration since the soft modules are designed for use by individuals with little or no muscular power. Figure 2 shows the silicone modules attached to a human participant.

Generally, both the actuator geometry and attachment should be designed to suit the geometry of the patient. In our case, a generic design was made for the purpose of experiments.

\section{CONTROL SCHEME}

The experimental system of pressure and angle measurement has four major parts (Figure 3): A webcam, computer for visual processing, pressure regulation system and the wedge-shaped rubber actuator. The webcam provides visual information, which is processed by an image processing software. The pressure regulation system consists of a pump (air and vacuum), by which pneumatic pressurization and de-pressurization can be obtained; inlet and exhaust solenoid valves; pressure sensor; and microcontroller.

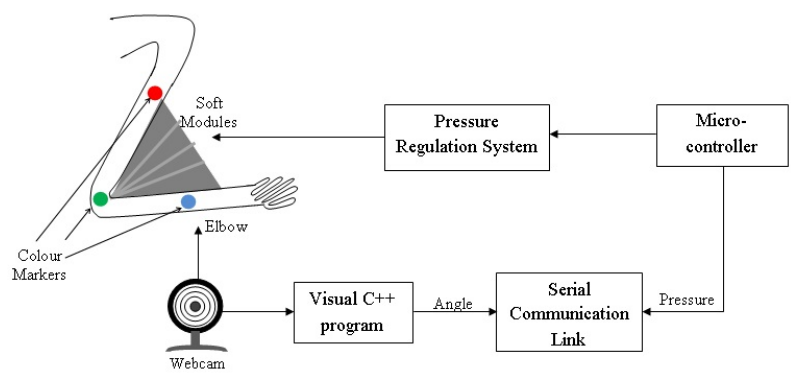

Fig. 3: Schematic picture of experimental system. The soft modules are attached to the elbow joint with red, blue and green colour markers positioned as shown, the Visual C++ program returns the angle of the elbow joint, the pressure sensor provides fedback by measuring the air pressure inside the modules.

\section{A. Visual Processing}

Visual processing was implemented in Visual C++ using OpenCV libraries. The processing was carried out in HSV (Hue-Saturation-Value) space. The video frames were preprocessed to filter and reduce noise. Red, Blue and Green colour markers were used to track the position of the upper arm and forearm. The centroid of each of the colour markers defined by their $\mathrm{x}$ and $\mathrm{y}$ coordinates were obtained as the 


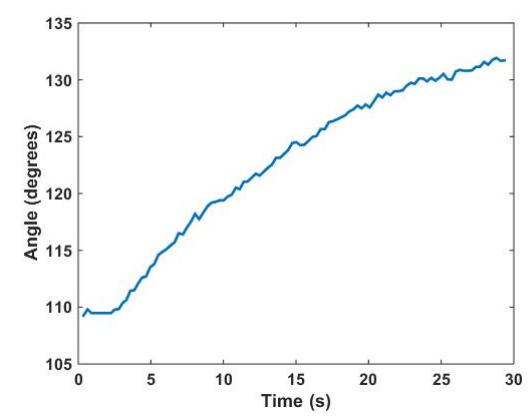

(a)

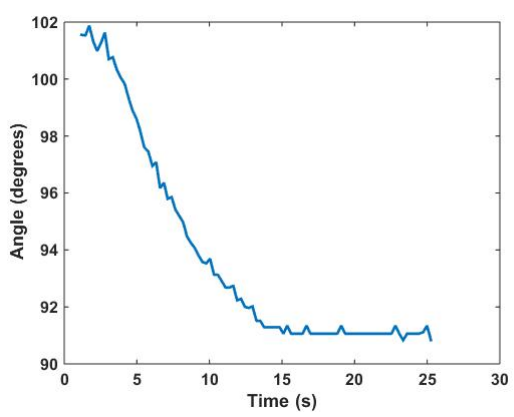

(b)

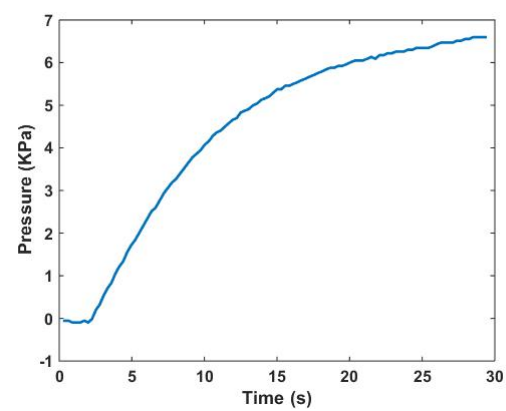

(c)

Fig. 4: Pressure of air and measured angle of extension/flexion of elbow while the soft modules were tested on a typical healthy test subject. The angle measurements were obtained via visual processing while the pressure data was obtained from readings from an analog gauge pressure sensor. a) Measured range of angle of elbow extension as a function of time. b) Measured range of angle of elbow flexion as a function of time. c) Pressure of air in the soft modules as a function of time while the modules performed extension motion.

position of the marker. Figure 5 shows the camera view of the image. The angle of the elbow is calculated as:

$$
q_{0}=\cos ^{-1}\left(\frac{l_{1}^{2}+l_{2}^{2}-l_{3}^{2}}{2 l_{1} l_{2}}\right)
$$

Where lengths $l_{1}, l_{2}$ and $l_{3}$ are calculated as the distance (in unit of pixels) between the centroid of the red and blue; blue and green; red and green colour markers respectively.

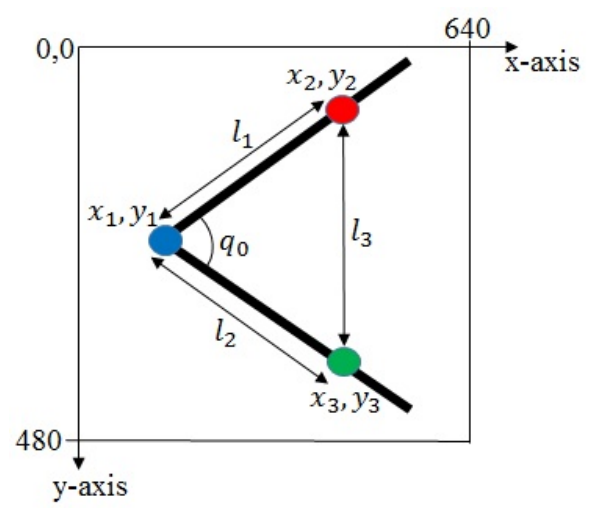

Fig. 5: The red, blue and green markers are positioned as indicated for measurement of the current elbow angle, $q_{0}$. The webcam projects a 2D image of the scene as shown. Each $x, y$ position represents the pixel co-ordinate $[x, y]$ of the center position of each colour marker.

\section{B. Interfacing the microcontroller with the visual processing program}

A serial communication link was established to obtain the current angle measurement $q_{0}$ from the Visual processing program to the microcontroller which in turn would generate an appropriate Pulse Width Modulated (PWM) signal that would actuate the pump and valves to control the air pressure into the soft modules. This communication pathway was established through the serial interface of the computer as shown in Figure 3.

\section{Pressure Regulation Circuit}

Figure 6 shows the arrangement of pumps and valves for the soft actuation of the modules. As shown, it consists of an air pump to act as the pressure source; two solenoid valves (one acts as an inlet valve while the other as an exhaust valve) and an amplified analog pressure sensor to measure the air pressure in the soft modules. The solenoid valves act to control the flow of air into and out of the soft modules. The electronic circuit consists of a PIC16F876A microcontroller running at a clock speed of $4 \mathrm{MHz}$ and transistors to enable the high current valves and pump to be controlled by the microcontroller as shown in Figure 7. The solenoid valves are controlled by 2 output compare modules configured for PWM-mode. As no operating system kernel is used, mainly interrupt sub-routines and parallel running subunits are used. This works very well for analog input of pressure signals.

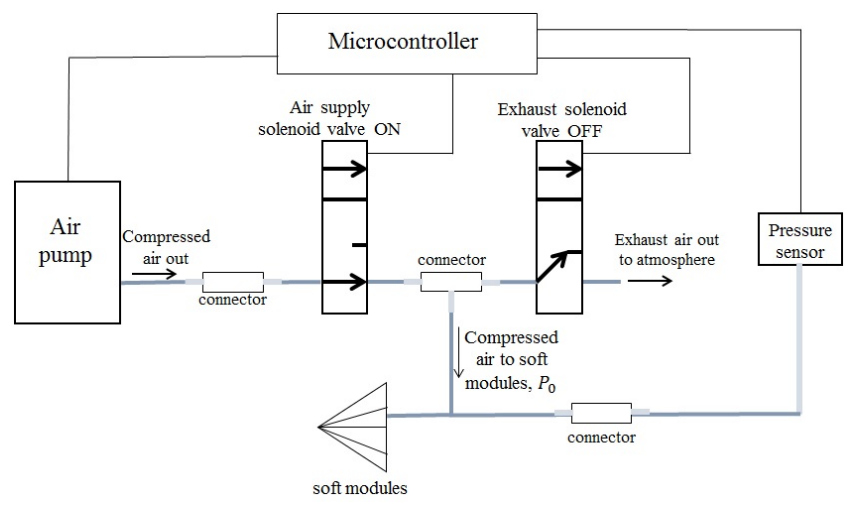

Fig. 6: Circuit arrangement of pressure controller. Figure shows the arrangement of the air-inlet and exhaust valves, pump, pressure sensor and soft modules.

Compressed air at a suppy pressure of $50 \mathrm{KPa}$ from the 
air pump passes through the air supply solenoid valve and changes to output pressure when the air supply solenoid valve turns ON. Therefore, air from the supply pump passes through the air supply solenoid valve and changes to output pressure. A PWM output is then produced on the output pin of the microcontroller to switch ON/OFF the exhaust valve in order to produce an output pressure equal to the desired pressure. The output pressure is fed back to the microcontroller via the pressure sensor. This is to check if the desired pressure, $P_{d}$, has become equal to the output pressure $P_{0}$. Pressure corrections then occur to produce an output pressure that is equal to the set pressure.

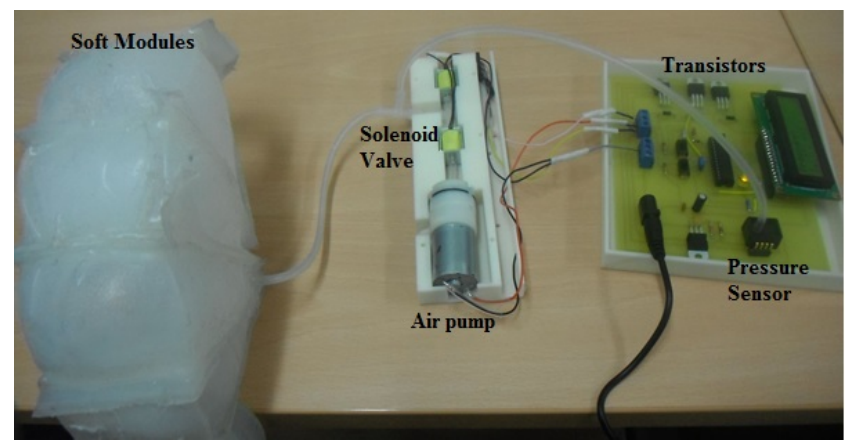

Fig. 7: Control circuit for the soft modules. Figure shows the soft modules, air-inlet and exhaust solenoid valves, air pump, transistors, pressure sensor and the PIC microcontroller.

\section{Joint Control}

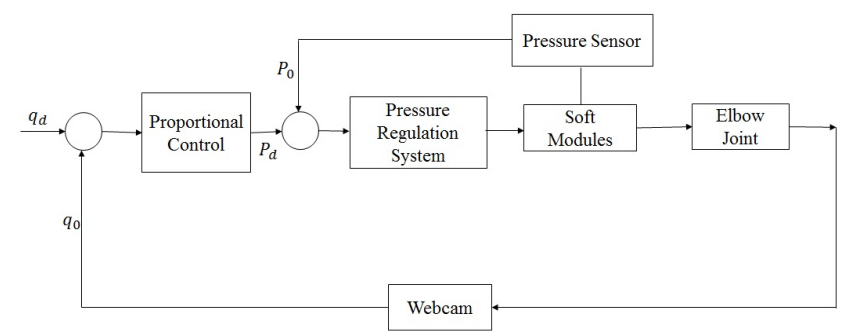

Fig. 8: Block Diagram of Joint Control. The lower pressure control loop uses information from the pressure sensor to get the output pressure, $P_{0}$ while the higher visual control receives information of the current angle of the elbow, $q_{0}$ from the webcam.

A controller for visual feedback provided by the webcam and pressure control are required for joint control. This visual-pressure control loop periodically receives discrepancies between the measured elbow angle $q_{0}$ and desired angle $q_{d}$ and uses a two-staged cascaded control approach to send a PWM signal to the pump and solenoid valves to resolve the difference. The time required for the computer vision program to process each frame was measured as $200 \mathrm{~ms}$.

The difference between the measured and the reference angle results in an error signal, which when passed to the proportional controller outputs a signal which is then used as setpoint to send the appropriate PWM signal to the solenoid valves to regulate the pressure in the soft actuator. Figure 8 shows the block diagram for the joint visual and pressure control. With this control method, open loop joint control is possible by varying the amount of desired pressure, $P_{d}$, that is added to the output of the control loop. The pressure feedback signal is provided by the means of a pressure sensor to measure pressure in the soft modules. The output of these inner pressure control corresponds to the duty cycle of the PWM signal that drives the solenoid valves. To measure pressure, amplified pressure sensors ( 0 - 5psi pressure range) with analog interface having a $1 \mathrm{~ms}$ response time was used. A 10-bit Analog-to-Digital Converter (ADC) resolution was used to read the pressure. Using the microcontroller PWM features, pressure is controlled by varying the length of the duty cycle of the air pump.

\section{METHODS}

A light weight anatomical model of an elbow joint made of swimming float and 3D-printed hinge joint was first used to test the use of these soft modules. The model was used as a first step in taking measurements. Thereafter healthy subjects were used to verify the use of the soft modules. A typical subject sits on a chair, puts on the soft modules, places the elbow on a table while exercises are carried out with pneumatic actuation. The assistive soft modules was tested on three healthy participants between the ages of $25 \pm 5$ yrs and body weight within the range of $65 \pm 10 \mathrm{~kg}$. The participants wore the soft modules for a duration of 15 minutes while actuation was carried out and measurement data were recorded. Figure 2 shows the modules rotating the elbow of a test subject.

\section{EXPERIMENTAL RESULTS}

The graphs for angle and air pressure measurements for a typical test participant is shown in Figure 4. The experiments were conducted by using the soft modules to actuate the elbow while pressure and angle measurements were recorded. The purpose of these experiments was to show that through a combination of the control (visual processing and microcontroller program) and design (that is the inherent softness, lightness and compliance of the soft modules), we can successfully accomplish the primary task of actuating an elbow joint for flexion and extension.

Figure $4 \mathrm{c}$ shows the pressure of air in the soft modules during actuation. Measurements of air pressure in the soft actuator was taken with an analog pressure sensor. When an individual triangle segment is inflated, there is a change in angle and also a bulge on the soft layer of the triangle. This is because the inflation pressure $P_{0}$ gives rise to extension both the circumferential and axial directions. This bulge reduced the efficiency at which the triangle modules increased in angle. If the bulge can be minimised, the angle change in each of the triangle segment would increase.

Using 6V DC supply to power the control circuit at a supply pressure of $50 \mathrm{KPa}$, and for a desired elbow flexion 
and extension motion between $70^{\circ}-110^{\circ}$, the measured maximum frequency of the soft modules is $0.03 \mathrm{~Hz}$. For this system, it can be difficult to achieve high accuracy of vision and pressure measurements. This is expected as a result of delay by the high-level visual control and also due to some parameters of the solenoid valve such as leakage and exhaust speed. Also, air leakage at the connecting point between the soft robot and the tubing; loose tubing connection between the pump, valves and pressure sensor will limit the accuracy of this system. In order to achieve a good computer vision measurement system, a recalibration of the HSV values of the three colour markers was done every time measurements were taken. This was done so as to accurately track the correct pixel coordinate of the colour markers each time the experiment was conducted, thereby minimising the effect of change in lighting conditions of the room and ambient light. The second area of performance evaluation is the joint output torque the soft modules can produce. The measured maximum torque achieved for this rehabilitation device is 2.7Nm.

\section{CONCLUSION}

A wearable assistive rehabilitation device for stroke patients or individuals with little or no power/muscle control in the elbow was designed. To achieve this, an entirely soft design constructed uniquely from only silicone rubber, with inherent low weight and safety was implemented. The ability of this entirely soft assistive arm to rotate an elbow joint was demonstrated, which leads to many potential applications such as providing muscle power, restoring motor functions and as a neuro-rehabilitation device. A microcontroller operated pressure regulator system to perform pneumatic actuation of the soft modules was also presented. A visual processing system to record the angle measurements of the elbow was presented. The system was designed to meet the specifications of low weight, ease of wearing and comparatively low mechanical complexity, which are essential for any device adapted for use on the human body. The performance of the soft modules suggests its application as a physiotherapy training device for elbow flexion and extension. The complete soft robotic device including the cost of the soft modules and actuation components is essentially low and therefore can be readily available to every member of the populace including developing countries.

\section{ACKNOWLEDGMENT}

The authors would like to thank The Great Britain Sasakawa Foundation (Project Number: 4391) for the international exchange, Dr. Guy Haworth for his support, Mr. Ben Haworth for his master thesis, Mr. Steve Gould and Mr. Nick Dove for their help in producing the control board.

\section{REFERENCES}

[1] W. S. Harwin, A. Murgia and E. K. Stokes, "Assessing the effectiveness of robot facilitated neurorehabilitation for relearning motor skills following a stroke", Medical and Biological Engineering and Computing, Springer 49 No.10, pp. 1093-1102, 2011.
[2] Y. Hayashi, K. Nagai, K. Ito, J. S. Nasuto, C.V. R. Loureiro and S. W. Harwin, "Feasible Study of EEG driven Assist Robot System for Stroke Rehabilitation", IEEE International Conference on Biomedical Robotics and Biomechatronics, pp. 1733-1739, 2002.

[3] S. Coote, B. Murphy, W. Harwin and E. Stokes, "The effect of the GENTLE/s robot-mediated therapy system on arm function after stroke", Clinical Rehabilitation, No.5, pp. 395-405, 2008.

[4] R. C. V. Loureiro and W. S. Harwin, "Reach \& grasp therapy: Design and control of a 9-DOF robotic neuro-rehabilitation system", International Conference on Rehabilitation Robotics, pp. 757-763, 2007.

[5] C. D. Takahashi, L. Der-Yeghiaian, V. H. Le, and S. C. Cramer, "A Robotic Device for Hand Motor Therapy After Stroke", IEEE International Conference on Rehabilitation Robotics, 2005.

[6] R. C. V. Loureiro, W. S. Harwin, R. Lamperd and C. Collin, "Evaluation of Reach and Grasp Robot-Assisted Therapy Suggests Similar Functional Recovery Patterns on Proximal and Distal Arm Segments in Sub-Acute Hemiplegia”, IEEE Transactions on Neural Systems and Rehabilitation Engineering, pp. 593-602, 2014.

[7] P. Polygerinos, S. Lyne, Z. Wang, F. L. Nicolini, B. Mosadegh, G. M. Whitesides and C. J. Walsh, "Towards a Soft Pneumatic Glove for Hand Rehabilitation", IEEE International Conference on Intelligent Robots and Systems, 2013.

[8] N. G. Tsagarakis and D. G. Caldwell, "Improved modelling and assessment of pneumatic muscle actuators", IEEE International Conference on Robotic and Automation, pp. 3641-3646, 2000.

[9] B. Tondu, P. Lopez, "Modeling and control of McKibben artificial muscle robot actuators", IEEE Control Systems, Vol. 20, Issue 2, 2002.

[10] Y. S. Song, Y. Sun, R. Brand, J. Zitzewitz, S. Micera, G. Courtine and J. Paik, "Soft Robot for Gait Rehabilitation of Spinalized Rodents", IEEE International Conference on Intelligent Robots and Systems, 2013.

[11] P. Maeder-York, T. Clites, E. Boggs, R. Neff, P. Polygerinos, D. Holland, L. Stirling, K. C. Galloway, C. Wee, C. J. Walsh, ”Biologically Inspired Soft Robot for Thumb Rehabilitation", Proceedings of Design of Medical Devices Conference, 2014.

[12] S. Schulz, B. Schmitz, R. Wiegand, C. Pylatiuk and M. Reischl, "The Hybrid Fluidic Driven Upper Limb Orthosis-OrthoJacket", Proceedings of the MyoElectric Controls/Powered Prosthetics Symposium Fredericton, 2011.

[13] A. D. Marchese, R. K. Katzschmann and D. Rus, "Whole Arm Planning for a Soft and Highly Compliant 2D Robotic Manipulator", IEEE International Conference on Intelligent Robots and Systems, pp 554 - 560, 2014.

[14] R. F. Shepherd, F. Ilievskia, W. Choia, S. A. Morina, A. A. Stokes, A. D. Mazzeoa, X. Chena, M. Wanga and G. M. Whitesides, "Multi-gait soft robot", PNAS, 2011.

[15] F. Ilievski, A. D. Mazzeo, R. F. Shepherd, X. Chen, and G.M. Whitesides, "Soft Robotics for Chemists", Angewandte Chemie, Vol. 123, pp. 1930 - 1935, 2011.

[16] R. V. Martinez, J. L. Branch, C. R. Fish, L. Jin, R. F. Shepherd, M. D. Nunes, Z. Suo and G.M. Whitesides, "Robotic Tentacles with Three-Dimensional Mobility Based on Flexible Elastomers", Advanced Materials, Vol. 25, pp 205 212, 2013.

[17] Y. Sun, Y. S. Song and J. Paik "Characterization of Silicone Rubber Based Soft Pneumatic Actuators", IEEE International Conference on Intelligent Robots and Systems, 2013.

[18] V. Oguntosin, S. J. Nasuto and Y. Hayashi, "A Compact Low-Cost Electronic Hardware Design for Actuating Soft Robots", UKSIMAMSS International Conference on Modelling and Simulation, pp. 242247, 2015.

[19] N. G. Kutner, R. Zhang, A. J. Butler, S. L. Wolf and J. L. Alberts, "Quality-of-life change associated with robotic-assisted therapy to improve hand motor function in patients with subacute stroke: a randomized clinical trial", Physical therapy, Vol. 90, pp. 493- 504, 2010.

[20] H. I. Krebs, B.T. Volpe, M.L. Aisen and N. Hogan, "Increasing productivity and quality of care: Robot-aided neuro-rehabilitation", Journal of Rehabilitation Research and Development, 2000.

[21] A. Gil-Agudo, A. Ama-Espinosa, A. Reyes-Guzmn, A. Bernal-Sahun and E. Rocon, "Applications of Upper Limb Biomechanical Models in Spinal Cord Injury Patients", Biomechanics in Applications, 2011. 